\title{
Linx
}

Revue des linguistes de l'université Paris X Nanterre

$54 \mid 2006$

La cause : approche pluridisciplinaire

\section{Sens et grammaire universelle : théorie et constats empiriques}

\section{Anna Wierzbicka}

Traducteur : Bert Peeters

URL : http://journals.openedition.org/linx/520

DOI : $10.4000 /$ linx.520

ISSN : 2118-9692

\section{Éditeur}

Presses universitaires de Paris Nanterre

\section{Édition imprimée}

Date de publication : 1 juin 2006

Pagination : 181-207

ISSN : 0246-8743

Référence électronique

Anna Wierzbicka, "Sens et grammaire universelle : théorie et constats empiriques », Linx [En ligne], 54 | 2006, mis en ligne le 01 août 2007, consulté le 01 mai 2019. URL : http://journals.openedition.org/ linx/520; DOl : 10.4000/linx.520 


\title{
Sens et grammaire universelle : théorie et constats empiriques ${ }^{1}$
}

\author{
Anna Wierzbicka \\ Australian National University
}

Traduit de l'anglais par Bert Peeters (University of Tasmania)

\section{Introduction}

Le titre de cette conférence - «Sens et grammaire universelle: Théorie et constats empiriques »- rappelle celui d'un ouvrage publié il y a deux ans par la maison John Benjamins, dans la Studies in Language Companion Series. Il s'agit d'un ouvrage coordonné par mon collègue Cliff Goddard et moi-même, et qui inclut, hormis nos propres chapitres, qui servent de toile de fond, des études détaillées - des portraits en quelque sorte, réalisés par des auteurs différents - de six langues géographiquement dispersées, de nature très divergente, et appartenant à des familles linguistiques tout à fait distinctes. On y trouve des chapitres sur le malais (Cliff Goddard), le mandarin (Hilary Chappell), le laotien (Nick Enfield), l'espagnol (Catherine Travis), l'une des nombreuses langues austronésiennes de la Nouvelle-Guinée (Robert Bugenhagen) et le polonais (Anna Wierzbicka).

L'objectif de l'ouvrage était d'établir le noyau linguistique que partagent toutes les langues, et ce par des moyens empiriques, c'est-à-dire en étudiant un certain nombre de langues dissemblables et en identifiant ce qu'elles partagent - du point de vue lexical aussi bien que grammatical. Au bout des dix ans ou presque que mon

\footnotetext{
${ }^{1}$ Le texte que voici correspond grosso modo à celui d'une conférence présentée au collège de France en février 2004. Une version antérieure et condensée figure dans les Cabiers Ferdinand de Saussure (vol. 60, 2006).
} 
Anna Wierz̧bicka

corédacteur et moi avons consacrés au projet qu'il couronne (voir p.ex. Goddard et Wierzbicka réd. 1994 ; Wierzbicka 1996, 1998 ; Goddard 1998, 2001), nous croyons largement avoir réalisé notre but. Ce que je vous propose aujourd'hui, c'est un résumé des constats les plus importants, classés dans trois rubriques: d'abord, le lexique universel ; ensuite, la grammaire universelle; et enfin, la «métalangue sémantique naturelle » (ci-après « MSN»). Le terme $M S N$, auquel je reviendrai plus loin, représente le noyau commun de toutes les langues du monde - une mini-langue universelle située au cœur de toutes les langues naturelles, qu'en tant que métalangue elle permet de décrire de façon éclairante.

\section{Le lexique}

Tout auteur de dictionnaires bilingues est conscient du fait qu'il faut s'attendre à ce que, d'une langue à l'autre, il y ait des différences sémantiques même entre des unités lexicales sémantiquement apparentées. Un grand nombre de mots anglais, par exemple, n'ont pas d'équivalents précis en français, et vice versa. Or, s'il en est ainsi quand le nombre de langues rapprochées s'élève à deux, que dire du postulat qu'il existe des mots qui, polysémie mise à part, ont des contreparties exactes dans toutes les langues, sinon qu'il est complètement fantaisiste ? Et pourtant, c'est exactement ce que l'étude empirique approfondie de nombreuses langues dissemblables, par des chercheurs différents, au cours des années, a permis de constater : à savoir qu'il y a effectivement des mots (ou bien des éléments comparables) qui ont des équivalents sémantiques exacts dans toutes les langues (ou du moins dans celles qui ont été examinées à ce sujet). Autrement dit, il a été possible, de façon empirique, de dresser une liste de mots, ou d'éléments comparables, possédant des équivalents sémantiques exacts dans un nombre de langues dissemblables, et de corroborer cette liste en élargissant l'échantillon de langues examinées. Au fur et à mesure de nos explorations, des contre-exemples se sont manifestés, voire ont été activement recherchés ; et toute découverte de contreexemples, pour peu qu'ils soient irréfutables, a conduit à une révision de la liste hypothétique de "mots universels» (ou d'éléments comparables universels). La liste telle qu'elle se présente à l'heure actuelle est, on l'aura compris, le résultat de recherches minutieuses, et ne contient aucun élément dont le caractère universel ait été valablement mis en cause.

La liste la plus récente comporte quelque soixante éléments, énumérés dans la Table 1 ci-dessous - une table qui n'est pas sans présenter certaines analogies avec la table des éléments chimiques de Mendeleïev. Je n'irai pas jusqu'à dire que cette liste divisée en groupes un tant soit peu thématiques - est absolument finale, mais il y a lieu de croire qu'il s'en faut de peu. Elle est l'aboutissement de recherches empiriques effectuées pendant plus de trois décennies par une multitude de chercheurs; et s'il est vrai qu'elle a subi maintes révisions au cours des années, il faut immédiatement ajouter que ces révisions, loin d'être arbitraires, représentent des approximations successives de l'objectif - un objectif qui, plutôt que de reculer à mesure qu'on cherche à s'en rapprocher, se précise de plus en plus. 
Table 1 : Table des primitifs conceptuels en anglais (cf. Wierzbicka 1996, Goddard 1998)

Substantifs :

Déterminants :

Quantificateurs :

Attributs :

Prédicats mentaux :

Discours :

Actions, événements, mouvement :

Existence et possession:

Vie et mort :

Concepts logiques :

Temps :

Espace :

Intensificateur, augmentateur :

Taxonomie, partonomie :
Similarité :

I, YOU, SOMEONE, SOMETHING, PEOPLE, BODY

THIS, SAME, OTHER

ONE, TWO, SOME, MUCH/MANY, ALL

GOOD, BAD, BIG, SMALL

THINK, KNOW, WANT, FEEL, SEE, HEAR

SAY, WORDS, TRUE

HAPPEN, MOVE

THERE IS, HAVE

LIVE, DIE

NOT, MAYBE, CAN, BECAUSE, IF

WHEN, NOW, AFTER, BEFORE, A LONG TIME, A SHORT TIME, FOR SOME TIME, IN ONE MOMENT

WHERE, HERE, ABOVE, BELOW, FAR, NEAR, SIDE, INSIDE, TOUCH

VERY, MORE

KIND OF, PART

LIKE

Les primitifs :

- se lexicalisent sous forme de mots, de morphèmes liés ou de phrasèmes ;

- ils peuvent être formellement, c'est-à-dire morphologiquement, complexes ;

- d'une langue à l'autre, ils peuvent avoir des propriétés morphosyntaxiques différentes (et notamment appartenir à des catégories lexicales distinctes) ;

- ils peuvent avoir des variantes combinatoires (des allolexes).

Je m'empresse d'ajouter que, considérée telle quelle, sans autre justificatif, la liste ci-dessus est susceptible d'induire en erreur, et ce pour plusieurs raisons. Un grand nombre des mots anglais qui y figurent sont polysémiques, mais il n'y a qu'un seul sens par mot (ou par phrasème) qui est présumé avoir une contrepartie sémantique exacte dans toutes les autres langues. Afin d'illustrer quel est le sens présumé lexicalisé dans toutes les langues, il est utile de recourir à ce que nous avons appelé des «contextes canoniques ». Le mot anglais move, par exemple, a plusieurs sens, qu'illustrent les trois phrases ci-après :
A : I can't move.
(Je ne peux pas bouger.)
B : $\quad$ That story moved me.
(Cette histoire m'a ému.)
C: $\quad$ They moved to another city.
(Ils se sont établis dans une autre ville.)

Des trois sens distincts de move illustrés dans ces phrases, c'est le premier qui est censé avoir des contreparties exactes dans toutes les langues du monde, et non pas le deuxième ni le troisième. En polonais, chacune des phrases $\mathrm{A}, \mathrm{B}, \mathrm{C}$ nécessiterait un verbe différent (et les traductions données démontrent clairement qu'il en est de même 
en français). Ce n'est que le premier de ces verbes qui est présumé représenter un concept humain universel, avec des lexicalisations dans toutes les langues.

L'universalité du concept BOUGER (le but des petites capitales est de distinguer le concept monosémique du mot polysémique) peut être testée dans un environnement de "phrases canoniques» telles que Je ne peux pas bouger ou bien Cette chose bouge, étant donné que les divers autres mots utilisés dans ces phrases appartiennent eux aussi à la liste hypothétique des universaux du lexique. Ainsi donc, ce qui est affirmé ici, c'est que, hormis BOUGER, les concepts MOI, POUVOIR, NE ... PAS, CE et CHOSE (QUELQUE CHOSE) ont des contreparties sémantiques exactes dans toutes les langues. En fait, nous soutenons que la phrase entière Je ne peux pas bouger peut se traduire dans n'importe quelle autre langue, sans la moindre altération sémantique ; et qu'il en est de même dans le cas d'autres phrases canoniques, p.ex. Cette chose bouge. En revanche, des phrases telles que Cette histoire m'a ému ou bien Ils se sont établis dans une autre ville ne sauraient se traduire de la même façon, sans altération sémantique, dans d'autres langues, puisque dans beaucoup d'autres langues il n'y a pas de mots (ni de morphèmes ni de phrasèmes) équivalant, du point de vue sémantique, au mot anglais move (dans les sens illustrés dans les phrases $\mathrm{B}$ et $\mathrm{C}$ ), ni à plus forte raison à des mots tels que story 'histoire' ou city 'ville'.

Il n’y a pas que l'existence de structures polysémiques linguistiquement uniques qui demande à être signalée et justifiée. Faute d'espace et de temps, je n'entrerai pas dans le détail, mais je renvoie aux chapitres pertinents de Meaning and Universal Grammar (Goddard et Wierzbicka réd. 2002 ; ci-après Sens et grammaire universelle). Je proposerai simplement quelques listes équivalentes à celles de la Table 1: une première pour le malais, l'une des langues étudiées en profondeur dans ledit ouvrage, et une seconde pour le français. Je répète, afin d'éviter tout malentendu, que les éléments de chacune de ces listes peuvent avoir des "allolexes », c'est-à-dire des variantes lexicales non énumérées ici; et aussi que, si les mots donnés dans les listes sont polysémiques, un seul sens est en cause.

Table 2 : Table des primitifs conceptuels en malais (cf. Goddard 2002a)

Substantifs :

AKA, KAU, SESEORANG, SESUATU, ORANG, BADAN

Déterminants :

INI, (YANG) SAMA, LAIN

Quantificateurs :

SATU, DUA, BEBERAPA, BANYAK, SEMUA

Attributs :

BAIK, BURUK, BESAR, KECIL

Prédicats mentaux : $\quad$ FIKIR, TAHU, MAHU, RASA, NAMPAK, DENGAR

Discours : $\quad$ KATA, PERKATAAN, BENAR

Actions, événements, mouvement : BUAT, TERJADI, BERGERAK

Existence et possession: $\quad$ ADA, ADA

Vie et mort :

HIDUP, MATI

Concepts logiques :

Temps :

TIDAK, MUNGKIN, BOLEH, SEBAB, KALAU

BILA, SEKARANG, SELEPAS, SEBELUM, LAMA, SEKEJAP, BEBERAPA LAMA, SAAT 
Espace :

(DI) MANA, (DI) SINI, (DI) ATAS, (DI) BAWAH, JAUH, DEKAT, (DI) SEBELAH, (DI) DALAM, TOUCH

Intensificateur, augmentateur :

SANGAT, LAGI

Taxonomie, partonomie :

JENIS, BAHAGIAN

Similarité :

MACAM

Table 3 : Table des primitifs conceptuels, version française (cf. Peeters réd. 2006)

Substantifs :

JE, TU, QUELQU'UN, QUELQUE CHOSE, GENS, CORPS

Déterminants :

CE, MÊME, AUTRE

Quantificateurs :

UN, DEUX, IL Y A ... QUI, BEAUCOUP, TOUT

Attributs :

BIEN, MAL, GRAND, PETIT

Prédicats mentaux :

PENSER, SAVOIR, VOULOIR, SENTIR, VOIR, ENTENDRE

Discours :

DIRE, MOTS, VRAI

Actions, événements, mouvement: FAIRE, ARRIVER, BOUGER

Existence et possession :

IL Y A, AVOIR

Vie et mort :

Concepts logiques :

VIVRE, MOURIR

Temps :

NE ... PAS, PEUT-ÊTRE, POUVOIR, À CAUSE DE, SI

QUAND, MAINTENANT, APRÈS, AVANT, LONGTEMPS, PEU DE TEMPS, POUR UN TEMPS, EN UN MOMENT

Espace :

OÙ, ICI, AU-DESSUS, AU-DESSOUS, LOIN,

Intensificateur, augmentateur : PRÈS, CÔTÉ, DANS, TOUCHER

Taxonomie, partonomie :

Similarité : TRÈS, PLUS

TYPE DE, PARTIE

COMME

Le mot primitifs dans l'en-tête des tables est important et demande à être expliqué. Si nous avons raison d'affirmer que les listes dressées ci-dessus pour trois langues différentes se correspondent, et se correspondent de façon rigoureuse, la question se pose de savoir pourquoi elles se correspondent.

Nous répondrons qu'il en est ainsi parce que les éléments dans ces listes constituent les composantes conceptuelles à l'aide desquelles se construisent toutes les idées humaines, dans tout ce qu'elles ont de plus complexe. Des cultures différentes peuvent certes produire des systèmes sémantiques différents, mais elles le font avec des configurations différentes des mêmes composantes conceptuelles - et celles-ci sont, dans tous les cas, données au départ. Dans le langage de philosophes du dixseptième siècle tels que Descartes et Leibnitz, ces composantes conceptuelles - ou " idées simples »- sont « innées »; dans le langage des sciences contemporaines, elles sont «câblées» dans le cerveau et font partie du patrimoine génétique humain. 
L'« innéité » ou le «câblage » des concepts humains correspondants n'est évidemment qu'une hypothèse. Mais l'universalité des éléments eux-mêmes, émergeant d'investigations empiriques, confère à cette hypothèse un haut degré de vraisemblance.

A une époque où le marché est inondé de nombreux ouvrages de vulgarisation témoignant de l'universalisme le plus brut qui soit - on pense particulièrement aux textes de Stephen Pinker (p.ex. Pinker 1994, 1997) - il faut dire et redire ce que les grands penseurs du passé (Locke, Humboldt, Saussure et d'autres) ont souvent souligné : à savoir qu'en règle générale les mots ne se correspondent pas sémantiquement d'une langue à l'autre. Il faut rejeter en même temps les formes extrêmes de relativisme : dans une perspective MSN, il convient de nuancer des assertions comme celle de Saussure (1971: 155) selon laquelle « il n'y a pas d'idées préétablies ».

Saussure a dit : «Si les mots étaient chargés de représenter des concepts donnés d'avance, ils auraient chacun, d'une langue à l'autre, des correspondants exacts pour le sens; or il n'en est pas ainsi » (1971: 161). C'est vrai en gros; trente années de recherches empiriques ont cependant permis d'établir que certains mots - par exemple ceux qui signifient VOIR, DIRE, VOULOIR, PENSER, BOUGER, FAIRE etc. - possèdent après tout, d'une langue à l'autre, des correspondants sémantiques exacts. C'est la raison pour laquelle nous professons - contre Saussure et de commun accord avec Descartes et Leibnitz - qu'il y a bel et bien des idées préétablies, à savoir nos quelque soixante primitifs universels. En fait, nous faisons nôtre la logique qui guidait le maitre de Genève. Il est vrai que des mots dont le signifié est indépendant du signifiant qui leur est associé dans une langue donnée doivent effectivement avoir des correspondants exacts dans d'autres langues. La prémisse saussurienne, cependant, était que des mots pareils n'existent pas - et la conclusion était qu'il n'y a donc pas d'idées préétablies. Nous savons mieux aujourd'hui, grâce aux recherches évoquées il y a quelques instants. Autrement dit, la prémisse était fausse, et il faut s'en réjouir : elle impliquait en effet l'impossibilité de comparer, de décrire et d'expliquer les sens lexicalisés dans des langues différentes, puisqu'il n'y avait pas de commune mesure. L'approche MSN, quant à elle, grâce à son ensemble empiriquement fondé de primitifs sémantiques universels, d'« idées préétablies » indépendantes des langues individuelles, dispose d'un outil qui lui permet de procéder à la description et à l'explication effectives du sens au-delà des frontières linguistiques et culturelles, description et explication dont la linguistique appliquée, y compris la lexicographie bilingue, l'enseignement des langues et la communication transculturelle, ne sauraient se passer.

On a pu reprocher à la théorie élaborée dans Sens et grammaire universelle et dans d'autres publications des corédacteurs et de leurs collègues d'être une tentative d'identification des universaux de la pensée et du langage humains par le moyen de mots anglais. Ce reproche repose sur un malentendu total. Ainsi que le montrent les tables ci-dessus, les concepts universaux proposés peuvent être identifiés à travers n'importe quelle langue. La liste anglaise fournit un moyen commode permettant de parler des primitifs dans des forums internationaux, mais l'anglais n'est choisi que par souci de commodité. Ce qu'il y a de plus important, c'est que des listes équivalentes peuvent être dressées pour toutes les autres langues, et que des phrases canoniques permettant de les mettre à l'épreuve des faits se laissent aisément construire dans toutes les langues. 
Le philosophe néerlandais Jaap Van Brakel fournit, du genre de critique auquel je viens de faire allusion, un exemple à la fois récent et illuminant. Je m'y attarderai quelque peu, car je crois qu'il est instructif. Le point de départ de Van Brakel (2002) est que les chercheurs qui publient en anglais adoptent souvent un certain nombre de concepts anglais, qu'ils mettent à profit dans la description d'autres langues, d'autres cultures et d'autres systèmes conceptuels. A son avis, le mot anglais colour 'couleur' est un exemple approprié d'un concept anglais transformé en instrument analytique : il a été utilisé, dit-il, pour décrire, dans toutes sortes de langues, dont certaines qui n'ont pas de mot correspondant, ce qu'on a coutume d'appeler le vocabulaire des couleurs. Van Brakel observe que le recours au mot anglais en vue de décrire l'univers conceptuel de locuteurs qui n'ont pas de mot signifiant 'couleur' dans leur propre langue constitue un exemple d'ethnocentrisme et donne à l'anglais du vingtième siècle une position privilégiée.

L'argument est valable, me semble-t-il ; en fait, je l'ai avancé moi-même dans trois études consacrées précisément à la sémantique de la perception visuelle (Wierzbicka 1990; 1996, chapitre 10; 1999; voir aussi Wierzbicka 1980: $42-44$ et 1985 : 77-80). Mais Van Brakel va plus loin : il fait valoir que tous les mots sont comme le mot anglais color (ou le mot français couleur), c'est-à-dire que tous les mots sont propres à la langue dont ils sont tirés, et qu'il n'y a pas de mots qui se correspondent à travers toutes les langues. A mon avis, c'est un postulat dogmatique et injustifié. En fait, des recherches empiriques portant sur une variété de langues donnent à croire qu'alors que la plupart des mots dans l'ensemble des langues sont effectivement comparables au mot couleur, et manquent d'universalité, certains ne le sont pas, et possèdent des contreparties sémantiques exactes dans toutes les langues. Les constats empiriques faits au sein de l'approche MSN permettent de dire que le mot voir est de ceux-là : si, effectivement, 'couleur' n'est pas à sa place dans la liste des concepts humains universaux, VOIR, quant à lui, en fait bel et bien partie, et se retrouve dans toutes les versions de la table des concepts humains universaux inclus dans Sens et grammaire universelle. L'universalité empiriquement établie de VOIR est par ailleurs la raison pour laquelle j'ai fait valoir, dans les trois études mentionnées il y a quelques instants (Wierzbicka 1990, 1996 et 1999), qu'au lieu d'étudier «la sémantique des couleurs» à travers les langues du monde, il serait plus approprié d'étudier «la sémantique de la perception visuelle ».

Van Brakel souscrit à mon refus de traiter 'couleur' et les soi-disant «couleurs élémentaires » ('blanc', 'noir', 'rouge', etc.) comme des instruments analytiques et des universaux présumés de la cognition humaine, mais il me reproche ensuite de traiter VOIR différemment, de le catégoriser parmi les universaux du lexique. Il me compte (p. 151) parmi les "défenseurs acharnés» de la thèse qu'il existe des universaux lexicaux et sémantiques, thèse qu'il rejette, et il poursuit en disant (je traduis) :

Elle [Wierzbicka] fait valoir que VOIR et quelques autres «éléments fondamentaux » sont des primitifs sémantiques universaux. Cependant, en voyant dans VOIR un élément primitif elle ne fait que répéter à un niveau plus abstrait l'erreur qu'elle dénonce de façon si éloquente pour COULEUR et COULEURS ÉLÉMENTAIRES. En me démarquant d'elle, je ne récuse pas la capacité de 'voir' dans la vie de tous les 
Anna Wierzbicka

jours, mais je récuse le primitif VOIR. Faire valoir que VOIR est un primitif sémantique revient toujours à 'donner à l'anglais du vingtième siècle une position privilégiée’ (Wierzbicka 1999).

Pourquoi Van Brakel s'obstine-t-il à croire que ni VOIR ni aucun autre concept ne saurait être un élément lexical ou lexico-grammatical réellement universel ? En dernière analyse, parce qu'il refuse d'accepter qu'il existe une méthode permettant d'établir que certains concepts se correspondent véritablement au-delà des frontières linguistiques ; et aussi parce qu'il est convaincu qu'il est facile de méprendre des quasi-équivalences pour des équivalences parfaites. Laissons de nouveau la parole à Van Brakel :

Wierzbicka souligne que la question des primitifs sémantiques est d'ordre empirique. Mais les questions empiriques ne sauraient être utilement posées que dans l'un ou l'autre cadre. Autre chose de dire que tous les peuples de la terre sont capables de voir (pourvu qu'ils ne soient pas aveugles), autre chose de dire que, toujours et partout, VOIR est un primitif sémantique. Il y a des dangers fondamentaux dans le postulat simpliste qu'il existe des primitifs sémantiques; que la traduisibilité des primitifs ne présente aucun problème inhérent; et que des mots ou des concepts anglo-américains permettent de les marquer.

Ces arguments valent la peine d'être examinés, et méritent d'être scrutés un par un. Il est vrai que je souligne que la question des primitifs sémantiques est d'ordre empirique : en faisant valoir que VOIR est universel, alors que 'couleur' ne l'est pas, je m'appuie sur des investigations empiriques qui ont montré qu'un grand nombre de langues n'ont pas de mot pour 'couleur', alors qu'à ce jour aucune langue n'a été identifiée qui n'ait pas de mot pour VOIR.

Je m'empresse d'ajouter que je suis tout à fait d'accord avec Van Brakel pour dire que les questions empiriques ne sont jamais purement empiriques, et qu'il nous faut un cadre théorique cohérent. Mais ce cadre, je le rappelle, existe... La théorie MSN, élaborée dans Sens et grammaire universelle et dans des publications moins récentes des corédacteurs et de leurs collègues, fournit en effet un cadre dans lequel des questions empiriques à propos des universaux lexico-grammaticaux peuvent être utilement posées, et au sein duquel différentes hypothèses soulevées par ces questions peuvent être mises à l'épreuve des faits.

Une autre assertion de Van Brakel qui ne me trouble pas le moins du monde est qu'il y a des «dangers fondamentaux dans le postulat simpliste qu'il existe des primitifs sémantiques» - mais on voit mal comment elle peut être adressée avec sérieux à une équipe de chercheurs qui, depuis des décennies, effectuent des recherches empiriques laborieuses et qui s'efforcent de trouver des contre-exemples invalidant leurs propres hypothèses de travail et de réviser ces hypothèses dès que des contre-exemples ont été repérés. Si cette démarche est «simpliste», on se demande comment décrire celle qui consiste à déclarer a priori qu'il est impossible d'identifier des universaux du lexique, s'évitant ainsi la peine d'explorer la question par des moyens empiriques, non pas pendant des années, mais pendant des décennies...

Passons à la mise en garde de Van Brakel contre le postulat «que la traduisibilité des primitifs ne présente aucun problème inhérent ». Il va sans dire - et 
on ne se lassera jamais de le répéter - que c'est faire preuve de simplisme de minimiser les problèmes de traduction et de postuler l'identité des sens là où elle n'existe pas. Il n'est pas moins vrai que la récusation de toute possibilité de traduction exacte témoigne d'un esprit stérile et dogmatique. Une fois de plus, la question est d'ordre empirique et, comme toute question empirique, ne peut être posée utilement qu'à condition qu'on dispose d'un cadre analytique approprié. Ceux qui se réclament de la théorie MSN ont à leur disposition un cadre qui répond à ces attentes.

Au sein de ce cadre, il est devenu coutumier de distinguer entre des phrases canoniques, composées exclusivement de concepts universaux hypothétiques, et des phrases « ordinaires». C'est qu'en reliant la question de la traduisibilité des primitifs putatifs au recours à des phrases canoniques, on peut procéder à des tests de vérification. Ainsi, dans le cas de VOIR, on peut avancer, parmi d'autres, les phrases canoniques que voici :

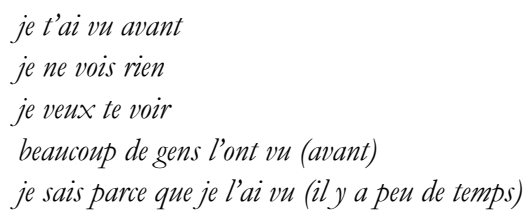

La traduisibilité de ces phrases a fait l'objet d'études extensives : aucune langue n'a été trouvée qui n'en permette pas de traduction exacte, c'est-à-dire dépourvue de différences sémantiques identifiables. Certes, il serait possible de critiquer le programme MSN en disant que des différences non détectées se cachent derrière des phrases canoniques de ce genre, soi-disant traduites de façon exacte dans les études détaillées rassemblées dans Sens et grammaire universelle. Seulement, de telles assertions, dogmatiques et aprioristes, seraient injustifiables et ne permettraient pas de faire avancer notre quête de la vérité ni d'assouvir notre désir de comprendre. En fait, elles ne manquent de frustrer cette quête et ce désir dans la mesure où elles obscurcissent délibérément la distinction entre des concepts manifestement non universaux tels que 'couleur' et des concepts manifestement universaux tels que VOIR.

En dénonçant le caractère illusoire des soi-disant «mots de couleurs élémentaires » et des soi-disant "émotions humaines fondamentales », Van Brakel a contribué de façon significative à démasquer les prétendus universaux de ces deux domaines. Cependant, son assaut du concept VOIR et, plus encore, son rejet aprioriste de l'existence de véritables universaux portent préjudice à sa contribution et apportent de l'eau au moulin de ceux qui se contentent d'utiliser l'anglais à titre de seule et unique métalangue analytique, ou bien qui s'y résignent.

Nous voilà prêts à répondre à la dernière des accusations qu'adresse Van Brakel aux dénicheurs de primitifs sémantiques universaux: "des mots ou des concepts anglo-américains permettent de les marquer ». J'espère que les trois tables présentées ci-dessus suffiront à lever ce malentendu; et que les six versions de la métalangue sémantique naturelle juxtaposées dans Sens et grammaire universelle le feront d'une manière encore plus spectaculaire, et plus efficace. Dans cet ouvrage, les primitifs sémantiques, plutôt que d'être exprimés en anglais, le sont en malais, mandarin, laotien, espagnol, polonais et mangaaba-mbula ; et les universaux de la grammaire le sont également. 
Anna Wierabicka

Van Brakel, qu'on dirait aussi hostile à l'idée qu'il y a des universaux de la grammaire qu'à l'idée qu'il y a des universaux du lexique, cite à l'appui de ses propres convictions Quine (1990), lequel, en traduction française, observe :

Il est par trop facile de faire l'impasse sur les critères behavioristes et de leur préférer des catégories personnelles imposées sans la moindre justification. Les universaux linguistiques, me semble-t-il, peuvent servir d'illustration. Un traducteur radical qui se débat avec une langue exotique sera naturellement et à juste titre amené, si la possibilité s'en présente, à la couler dans le moule de concepts grammaticaux familiers ; il ira même jusqu'à les adapter un peu, et le cas échéant il manipulera des terminologies familières. Ce sont des tendances de ce genre qui ont facilité la traduction au cours des âges et qui sont probablement largement responsables de l'apparente similarité structurelle d'une langue à l'autre. Si, dans une perspective d'ensemble, une construction quelconque frappe le linguiste comme étant universelle, qu'il examine ses critères. Seuls les critères behavioristes, applicables avant les directives des manuels de traduction, doivent faire conclure à l'universalité proprement dite.

Les thèses de Quine, l'une relative au recours à des «catégories personnelles imposées [à d'autres langues] sans la moindre justification ", l'autre relative aux faux universaux de la grammaire dérivés de la première langue du chercheur, sont évidemment tout à fait justes (quoiqu'elles manquent d'originalité). En revanche, la thèse de l'importance des "critères behavioristes » est si manifestement obsolète qu'à l'aube du $21^{\mathrm{e}}$ siècle plus personne ne pense sérieusement à la discréditer. Il me semble que la théorie MSN fournit une approche bien plus solide: elle s'appuie sur des critères sémantiques plutôt que behavioristes, et elle attache la plus grande importance à la vérification, par des locuteurs natifs, des hypothèses proposées.

\section{Grammaire}

La grammaire universelle est la deuxième des trois « rubriques » mentionnées dans l'introduction. Je commencerai par dire que l'idée de base a d'ores et déjà été présagée dans le volet précédent : j'ai fait valoir qu'il y a dans toutes les langues du monde des contreparties sémantiques exactes, non seulement d'éléments lexicaux comme bouger, moi, pouvoir, ne ... pas, ce et chose (quelque chose) mais aussi de phrases telles que Je ne peux pas bouger et Cette chose bouge. Cela veut dire que ce ne sont pas seulement les éléments eux-mêmes mais aussi les propriétés combinatoires qui sont universels. Autrement dit, dans toutes les langues du monde, le primitif MOI peut se combiner avec le primitif POUVOIR, le primitif POUVOIR avec le primitif NE ... PAS, et le primitif BOUGER avec le primitif POUVOIR et avec le primitif (QUELQUE) CHOSE. Certes, les configurations encodées lexicalement, phraséologiquement ou grammaticalement dans des langues différentes sont propres à ces langues - mais les règles de combinaison sont les mêmes dans toutes les langues. Il est possible, ainsi que nous l'avons démontré dans Sens et grammaire universelle, d'énumérer les possibilités combinatoires de chacun des primitifs. Il s'ensuit que chaque primitif conceptuel a sa propre grammaire 
conceptuelle, et que celle-ci est aussi universelle que le primitif lui-même. Ainsi, le primitif DIRE permet, de façon universelle, les options valentielles «allocutaire » et "sujet locutionnaire ", de sorte qu'il est possible d'exprimer, dans n'importe quelle langue, des sens équivalents à ' $\mathrm{X}$ a dit quelque chose à $\mathrm{Y}$ ' et ' $\mathrm{X}$ a dit quelque chose de Z' (même si le marquage formel de ces options valentielles différera d'une langue à l'autre). Autre exemple, d'un genre différent: le primitif SI apparaît universellement dans des phrases bipropositionnelles du type 'si tu fais cela, il pourra t'arriver du mal' (même si, dans certaines langues, la lexicalisation de SI coïncide, dans certains contextes, avec celle de QUAND).

La grammaire combinée de tous les primitifs constitue l'alpha et l'oméga de la grammaire universelle; les primitifs eux-mêmes, avec leur propriétés combinatoires, forment un système où - selon la célèbre formule d'Antoine Meillet - «tout se tient », et ce système, quant à lui, constitue le noyau conceptuel indivisible de toutes les langues humaines. En fait, l'idée fut présagée il y a plus de six cents ans, quand Roger Bacon professa que «la grammaire est, fondamentalement, la même dans toutes les langues, même s'il y a des différences superficielles » ("Grammatica una et eadem est secundum substantiam in omnibus linguis, licet accidentaliter varietur »). Qu'est-ce qui porta Bacon à s'exprimer de la sorte ? Essentiellement, la croyance que les fondements de la grammaire trouvent leur origine dans les fondements de la pensée humaine, et que ceux-ci sont partagés par tous les humains et par toutes les langues. C'est la conception de la grammaire universelle que les siècles ont consacrée, mais qui, jusqu'il y a peu, se trouvait largement repoussée par l'approche chomskyenne, une approche structurale de la grammaire universelle où le sens ne jouait aucun rôle réel. D'un point de vue historique, le programme poursuivi dans Sens et grammaire universelle se présente comme un retour à la tradition - quoiqu'il y ait une différence cruciale, à savoir l'analyse détaillée et rigoureuse des langues naturelles. Au bout de trente ans, le programme de recherches sémantiques inauguré en 1972 dans mon ouvrage Semantic Primitives, revitalisé ensuite, aux années quatre-vingts, par Cliff Goddard (cf. Goddard 1986, et 1989a et b) et continuellement amplifié au cours des deux dernières décennies grâce à de nombreux travaux effectués par Goddard et par moi-même, et aussi par d'autres collègues, a des contours suffisamment précis pour qu'il soit possible de faire un portrait détaillé et concret de ce qui fait l'unité de toutes les grammaires; c'est-àdire de déterminer où s'arrête ce qui est constant et où commence ce qui est variable, où s'arrête ce qui est essentiel et où commence ce qui est «accidentel », où s'arrête l'universel et où commence ce qui est propre à telle ou telle langue.

Dans Sens et grammaire universelle, Goddard et moi entrons dans le détail du modèle de grammaire universelle que nous proposons ; nous y précisons les propriétés syntaxiques inhérentes des primitifs sémantiques universaux. Faute de temps, je dois me contenter ici d'une présentation relativement sommaire de deux types de syntagmes que, par souci de commodité, j’appellerai «syntagmes substantivaux» et «syntagmes prédicatifs ». Pour un traitement plus approfondi, on verra Goddard et Wierzbicka (2002). 
Anna Wierzbicka

\subsection{Syntagmes substantivaux}

Un syntagme substantival se compose soit d'un substantif simple (MOI, TOI, QUELQU'UN / PERSONNE, GENS, QUELQUE CHOSE / CHOSE), soit de la combinaison d'un substantif et d'autres éléments qui, ensemble, forment une unité sémantique équivalente à un syntagme nominal (quoique non pas nécessairement identique, d'un point de vue structural, au syntagme nominal de l'anglais). Les substantifs se combinent avec des attributs (BIEN, MAL, GRAND, PETIT) dans ce qu'on pourrait appeler la « relation attributive ». On a donc des syntagmes substantivaux du type :

quelqu'un de bien, quelque chose de bien (du bien), des gens bien, des choses bien

quelqu'un de mal, quelque chose de mal (du mal), des gens méchants

quelque chose de grand, quelque chose de petit, de grandes choses, de petites choses

où $d u$ bien et $d u$ mal sont comme des «mots-valises » composés sémantiquement des primitifs QUELQUE CHOSE, BIEN et MAL, et utilisés avec certains prédicats (p.ex. PENSER) mais pas avec d'autres (p.ex. SENTIR).

La façon précise dont se réalise la relation attributive (par simple juxtaposition ou bien par d'autres moyens formels) diffère selon les langues; celles-ci se distinguent en outre selon que les mots attributifs appartiennent à une seule catégorie lexicale distincte (la classe des adjectifs) ou à plusieurs. Enfin, l'extension de ces constructions de type attributif à d'autres mots non inclus dans l'échantillon ci-dessus varie également d'une langue à l'autre.

Il y a d'autres relations, à part la relation attributive. Les substantifs catégoriels QUELQU'UN et QUELQUE CHOSE peuvent occuper la tête d'un syntagme substantival comportant le primitif CE / CELA ; on peut parler à cet égard de la «relation déterminative ». D'autres déterminants (MÊME, AUTRE) et d'autres quantificateurs (UN, DEUX, TOUT, BEAUCOUP, IL Y A ... QUI) peuvent eux aussi se combiner avec un substantif tête de syntagme QUELQU'UN ou QUELQUE CHOSE. Le primitif GENS possède des propriétés combinatoires semblables, mais plus limitées. Les listes cidessous fournissent des exemples simples de la relation déterminative, lexicalisés dans toutes les langues du monde.

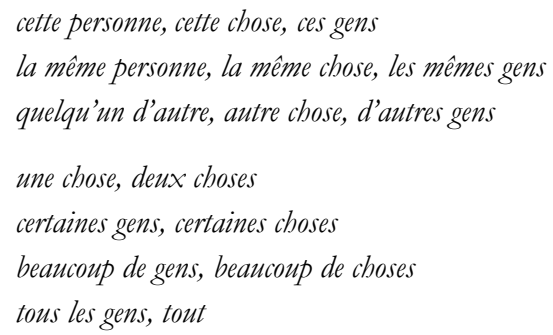

Je rappellerai que personne et chose sont des allolexes combinatoires de QUELQU'UN et de QUELQUE CHOSE, respectivement, et j'ajouterai que tout (= toutes les choses) est un "mot-valise » (comparable aux syntagmes $d u$ bien et $d u$ mal ci-dessus). En outre, en français, les combinaisons de QUELQUE CHOSE / CHOSE avec des quantificateurs tels que DEUX et BEAUCOUP sont soumises à une règle d' " accord en 
nombre » qui impose l'usage de la forme choses (avec $-s$ pluriel) au lieu de chose. De la même façon, CE prend la forme cette en combinaison avec CHOSE et PERSONNE, et ces en combinaison avec GENS. Ce sont là des instances d'allolexie propres au français.

Passons aux primitifs TYPE DE et PARTIE ; ce sont en quelque sorte des quasisubstantivaux, dans la mesure où, en combinaison avec des spécificateurs (c'est-à-dire des déterminants ou des quantificateurs), ils forment des syntagmes substantivaux. On a ainsi des syntagmes tels que :

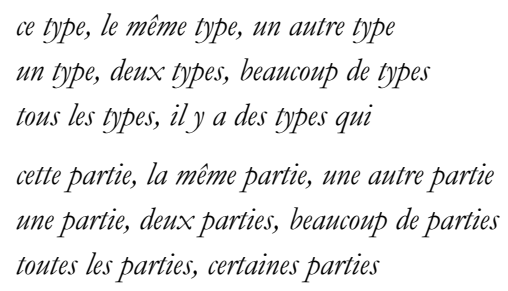

Les primitifs QUAND / MOMENT et OÙ / ENDROIT, quant à eux, forment également des syntagmes substantivaux, en combinaison avec des déterminants. OÙ / ENDROIT se combine en toute vraisemblance avec GRAND et PETIT.

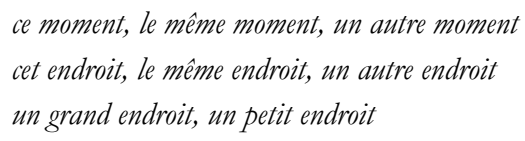

Enfin, tous les substantifs se laissent modifier par la combinaison COMME ÇA (= COMME + CE / CELA) :

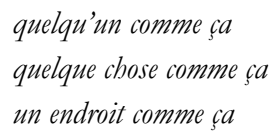

\subsection{Syntagmes prédicatifs}

Les éléments susceptibles de fonctionner comme prédicats sont de nature très diverse, et se laissent grouper de plusieurs façons différentes, selon leurs propriétés combinatoires. Toujours est-il que l'ensemble des prédicats se combinent avec NE ... PAS (négation), qui, de ce fait, a parfois été désigné dans la littérature MSN comme un «méta-prédicat». En outre, toute proposition, quel que soit le type dont il relève, peut inclure un adjoint temporel tel que MAINTENANT ou À CE MOMENT-LÀ. Il n'est pas sans intérêt de noter que la présence d'un tel adjoint (explicite ou bien sous-entendu) est indispensable avec certains prédicats. On peut voir dans ces prédicats - ARRIVER, MOURIR, BOUGER, FAIRE, VOIR, ENTENDRE, DIRE - l'équivalent de verbes prototypiques. À titre d'exemple, voici un échantillon de phrases canoniques relativement simples :

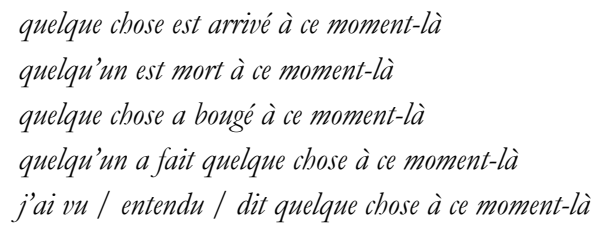


Anna Wierzbicka

Certains prédicats comportent deux parties, à savoir la partie principale, c'est-àdire le prédicat stricto sensu, et une partie subsidiaire, laquelle peut être désignée comme un « complément». Illustrons à l'aide des primitifs FAIRE, DIRE, VOULOIR et VOIR :

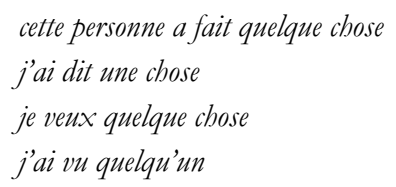

Précisons ensuite que la relation entre le mot prédicatif (FAIRE, DIRE, VOULOIR, VOIR...) et ses compléments (QUELQUE CHOSE, UNE CHOSE, QUELQU'UN...) diffère de celle qui existe entre la tête et le spécificateur au sein d'une relation attributive, ne fût-ce que parce qu'une tête peut normalement apparaitre avec ou sans son attribut, alors que le complément de prédicats comme FAIRE, DIRE, VOULOIR, VOIR etc. est obligatoire (à moins d'être omis par ellipse). En outre, il est clair que le rapport de dépendance va du complément au prédicat, plutôt qu'en sens inverse, puisque c'est le prédicat qui détermine si un complément est possible ou non, et quels sont les compléments qu'il admet. Ainsi, VOIR se combine de façon universelle avec les compléments QUELQUE CHOSE, QUELQU'UN et LES GENS, alors que DIRE et FAIRE (auxquels, dans plusieurs langues, il faut ajouter VOULOIR) n'admettent comme complément que le seul primitif QUELQUE CHOSE.

Le cas des prédicats MOURIR et VIVRE est différent. Il convient de noter que s'il est possible de traduire de façon exacte, dans toutes les langues du monde, des phrases canoniques telles que

$$
\begin{aligned}
& \text { Qu'a-t-il fait? } \\
& \text { Qu'a-t-il dit? } \\
& \text { Qu'a-t-il vu? }
\end{aligned}
$$

les prédicats MOURIR et VIVRE, quant à eux, n'admettent guère des structures de ce type. Cela signifie que FAIRE, DIRE et VOIR - contrairement à MOURIR et VIVRE disposent d'un «créneau» pour un syntagme substantival supplémentaire. Reste à savoir à quel(s) niveau(x) de représentation le prédicat (FAIRE, DIRE, VOIR, etc.) et le syntagme substantival (QUELQUE CHOSE, etc.) sont « indissociables ». Ce qu'il importe de souligner ici, c'est que, d'un point de vue sémantique, des expressions telles que faire quelque chose ou voir quelque chose forment des unités incontestables, indépendamment de la question de savoir si elles doivent en outre être considérées comme des constituants syntaxiques.

\subsection{Faire et arriver}

Regardons de façon quelque peu plus détaillée quelques-uns des primitifs prédicatifs et les propriétés syntaxiques qui leur sont attribuées au sein de la théorie MSN. Certains primitifs sémantiques ont ce que nous avons appelé plus haut des options valentielles (à ne pas confondre avec les « créneaux » obligatoires dont il vient d'être question). Par exemple, en plus de son sujet et de son complément (obligatoires), le primitif FAIRE peut prendre un substantif « patient». Du point de vue 
de leur réalisation en anglais, FAIRE et FAIRE À sont des instances d'un seul primitif. Le primitif FAIRE accepte en outre une option valentielle « instrumentale » et une option valentielle « comitative ».

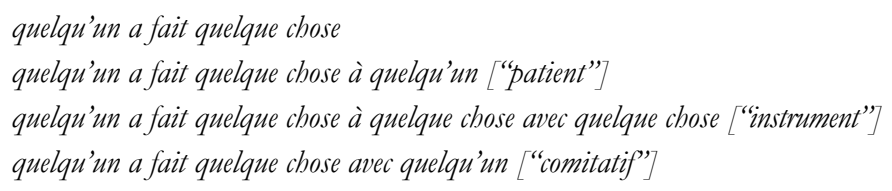

De façon plus ou moins analogue, le primitif ARRIVER admet à titre optionnel un substantif « objet affecté » (ARRIVER À), et aussi un substantif « locatif » (ARRIVER DANS CET ENDROIT).

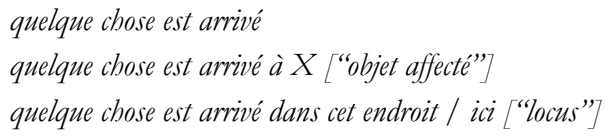

\subsection{Savoir, penser et dire}

Hormis leur préférence pour des substantifs personnels (MOI, TOI, QUELQU'UN, GENS) en position de «sujet», les primitifs SAVOIR et PENSER partagent un certain nombre d'options valentielles et de possibilités combinatoires dignes d'être relevées. Nous postulons qu'ils admettent l'un et l'autre un complément propositionnel (savoir que $P$ et penser que P), et qu'ils admettent en outre un "thème » (savoir de Y et penser à / de Y). Ils peuvent tous deux être suivis d'un complément substantival (savoir quelque chose, penser quelque chose). Enfin, PENSER peut prendre un complément « direct » d'ordre propositionnel, qui n'est pas sans rappeler celui que prend le prédicat DIRE (et auquel nous reviendrons dans quelques instants) :

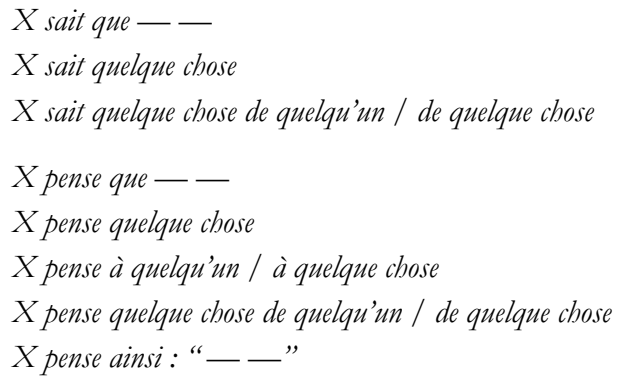

Par ailleurs, à l'instar de SAVOIR et de PENSER, avec lesquels il présente des affinités syntaxiques frappantes, DIRE peut prendre un «thème » optionnel (DIRE DE). En outre, il admet un complément substantival (à la façon de SAVOIR) et un complément « direct» (à la façon de PENSER). Il diffère cependant de tous les autres primitifs en ce qu'il possède une option valentielle distinctive pour l'allocutaire (DIRE À). On s'attend aussi à ce que DIRE se combine de l'une ou l'autre façon avec MOTS.

$X$ a dit quelque chose

$X$ a dit quelque chose de quelque chose 
Anna Wierzbicka

$$
\begin{aligned}
& X \text { a dit : "—_- } \\
& X \text { a dit quelque chose à quelqu'un } \\
& X \text { a dit ces mots }
\end{aligned}
$$

Il va sans dire que la réalisation formelle de ces différentes options valentielles et de ces différentes possibilités de complémentation différera d'une langue à l'autre. En anglais, par exemple, les arguments "thématiques» de DIRE, PENSER et SAVOIR sont formellement marqués de la même façon, à savoir la préposition about (SAY ABOUT 'dire de', THINK ABOUT 'penser à / de', KNOW ABOUT 'savoir de'). En français, par contre, PENSER se construit d'ordinaire avec $a ̀$; en revanche, si un complément substantival accompagne le «thème», celui-ci est introduit par de. On ne doit s'attendre ni à une distribution identique des marques formelles dans toutes les langues du monde, ni même au recours obligatoire à une adposition. Autre exemple : en mangaaba-mbula (Bugenhagen, dans Goddard et Wierzbicka réd. 2002, vol. 2, ch. 1), il n'y a qu'une seule préposition oblique générale, à savoir $p a$. Les exemples qui suivent montrent que pa est la marque du thème locutionnaire aussi bien que de l'allocutaire (que seul l'ordre des mots permet de distinguer entre eux).

mangaaba-mbula :

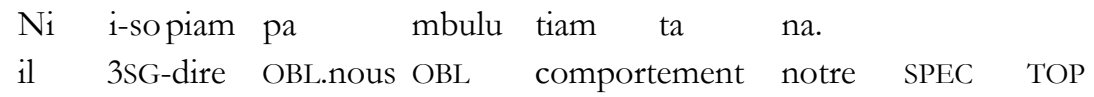

'Il nous a parlé au sujet de notre comportement.'

\section{La métalangue sémantique naturelle (MSN)}

Dans le programme de recherches qui a donné lieu à la publication de Sens et grammaire universelle, les deux parties du titre ne doivent pas être construites indépendamment l'une de l'autre : il n'y a pas - loin s'en faut - d'un côté le domaine du sens et de l'autre celui de la grammaire universelle. Dans notre programme, le sens et la grammaire universelle sont inséparables. Si, par souci de commodité et de brièveté, il nous arrive de décrire les quelque soixante éléments que nous appelons primitifs comme étant de nature « lexicale », il faut bien se dire que leur véritable statut est lexico-grammatical. Dans chacune des langues du monde, chacun des éléments a sa propre «incarnation » lexicale (phonologique), à moins d'en avoir plusieurs, ce qui arrive assez souvent, plus en particulier quand il y a des variantes lexicales - ou, dans la terminologie que nous avons adoptée, des «allolexes ». En même temps, chacun des éléments possède son propre ensemble de propriétés grammaticales (combinatoires).

Strictement parlant, le sens n'appartient point aux éléments individuels; il relève plutôt de mini-phrases, construites en combinant certains des éléments en fonction de leurs propriétés combinatoires universelles. Il n'y a que les phrases qui sont porteuses de sens, car seules des phrases sauraient exprimer et véhiculer des messages. La phrase Je ne peux pas bouger, construite à partir de primitifs conceptuels d'après les règles de la grammaire conceptuelle, est porteuse de sens, alors qu'un élément tel que BOUGER ne l'est pas, puisque tout seul il ne saurait véhiculer un message. Ainsi, le « sens » n'est pas, dans notre théorie, une entité assignée au lexique, 
ce qui réduirait la grammaire à un ensemble de «structures syntaxiques» formelles dépourvues de sens. Au contraire, nous l'avons déjà dit, les primitifs universels et l'ensemble universel de leur propriétés combinatoires constituent un seul système sémantique indivisible - un système que l'on considérera de préférence comme une mini-langue qui se prête de façon efficace, non seulement à la poursuite d'objectifs académiques (particulièrement dans le cas de linguistes qui s'adressent à d'autres linguistes), mais aussi à la communication humaine au-delà des cercles académiques.

Je me permettrai de fournir un seul exemple, traduit à moitié - à savoir l'ensemble de définitions proposé par Ray Jackendoff (1990:141) pour les mots d'émotion anglais to please 'plaire à', to displease 'déplaire à', to like 'aimer bien' et to fear 'craindre' (ou to bate 'détester') :

\begin{tabular}{|c|c|}
\hline $\mathrm{X}$ pleases $\mathrm{Y}$ & [état $\mathrm{AFF}+([\mathrm{X}],[\mathrm{Y}])]$ \\
\hline $\mathrm{X}$ displeases $\mathrm{Y}$ & [état AFF-[X], $[\mathrm{Y}] \mathrm{]}]$ \\
\hline Y likes X & [état RÉACT+ $([\mathrm{I}],[\mathrm{X})]$ \\
\hline Y fears/hates X & [état RÉACT-(IY], $[\mathrm{X}])]$ \\
\hline
\end{tabular}

La différence entre des systèmes artificiels de représentation tels que celui-ci et la métalangue sémantique naturelle, qui recourt à des mots tels que BIEN et MAL, c'est que ceux-ci existent dans toutes les langues et sont intuitivement intelligibles, alors que des plus et des moins ne le sont pas; et en outre, que des constructions syntaxiques simples telles que cela est mal ou bien quelque chose de bien est arrivé à cette personne (qui existent dans toutes les langues) sont elles aussi intuitivement intelligibles, alors que des crochets rectangulaires et des symboles tels que «AFF » ne le sont pas (Jackendoff est obligé de préciser que «AFF » signifie « entité affectée », et «RÉACT » « réaction »).

Mon objectif, ici, n'est pas de condamner les formules sémantiques qu'utilise Jackendoff. Je signalerai simplement que les formules sémantiques construites dans la métalangue sémantique naturelle sont radicalement différentes, et que c'est à cette différence que renvoie le mot naturel dans le nom de la métalangue : quoique cette métalangue ne soit pas une langue naturelle au sens où l'anglais, le français ou le malais sont des langues naturelles, il s'agit d'une métalangue "taillée » dans une langue naturelle (n'importe laquelle, par exemple l'anglais, le français ou le malais) et de ce fait intelligible à travers une langue naturelle (par exemple l'anglais, le français ou le malais).

La métalangue sémantique naturelle (MSN) qui utilise ces quelque soixante concepts universaux à titre de primitifs est un système formel, décrit en détail dans notre ouvrage Sens et grammaire universelle (voir aussi Wierzbicka 1996, et Goddard 1998). Ce n'est pas une langue naturelle; c'est l'équivalent d'un fragment de langue naturelle (n'importe laquelle). Son plus grand mérite est d'être assez proche de toutes les langues naturelles pour être intrinsèquement intelligible aux locuteurs de celles-ci.

Étant intelligible à travers des langues naturelles réelles, telles que l'anglais ordinaire ou le français ordinaire, la métalangue sémantique naturelle peut rendre service dans la communication humaine, l'enseignement des langues, la lexicographie et quantité d'autres domaines. On pourra l'utiliser en vue d'expliquer à des locuteurs ordinaires ce que signifie tel mot, ou telle expression, voire tel texte entier; on pourra s'en servir aussi afin d'expliquer des idées, voire d'entières idéologies en matière de droit, de religion, de politique, etc. (Voir, par exemple, mon ouvrage What did Jesus 
Anna Wierzibicka

mean? Explaining the Sermon on the Mount and the Parables in simple and universal human concepts, publié en 2001.)

La différence cruciale entre la MSN et d'autres systèmes de représentation sémantique est que ces autres systèmes sont le plus souvent, si on me permet le mot, «bilatéraux»: ils mettent des expressions linguistiques en rapport avec des représentations sémantiques quelconques, sans se soucier de ce que ces dernières apportent au locuteur ou à l'allocutaire. En comparaison, les explications rédigées en métalangue sémantique naturelle sont pour ainsi dire non pas bilatérales, mais quadrilatérales : d'une part, elles mettent des expressions linguistiques en rapport avec leurs représentations sémantiques; d'autre part, elles cherchent à expliquer ce que pourrait vouloir dire un locuteur qui se sert d'une expression quelconque, et à rendre compte de la façon dont un allocutaire pourrait interpréter ce qu'il vient d'entendre. Autrement dit, elles relient quatre entités plutôt que deux : un locuteur hypothétique, une expression linguistique, une représentation sémantique, et un allocutaire potentiel.

Voici un exemple - une explication (simplifiée) du mot allemand Schadenfreude, terme d'émotion emprunté tel quel en anglais (et qu'on entend parfois aussi en français), formulée à l'aide de concepts humains universels et d'une syntaxe universelle, d'abord en anglais, ensuite en allemand, et enfin en français :

En anglais : X felt Schadenfreude $=$

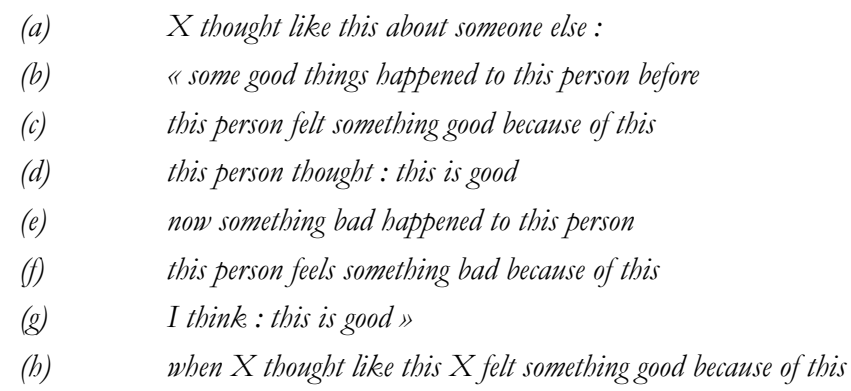

En allemand : $X$ füblte Schadenfreude $=$
(a) $X$ dachte so über jemand anders :
(b) "vorher sind dieser Person manche gute Dinge passiert
(c) diese Person füblte deswegen etwas Gutes
(d) diese Person dachte : das ist gut
(e) jetz̧t ist dieser Person etwas Schlechtes passiert
(f) diese Person füblt deswegen etwas Schlechtes
(g) ich denke : das ist gut 》
(b) als $X$ so dachte füblte $X$ deswegen etwas Gutes

Et en français : $X$ a senti de la schadenfreude $=$
(a) X a pensé comme ça de quelqu'un d'autre :
(b) "ily a des choses bien qui sont arrivées à cette personne avant
(c) cette personne a senti quelque chose de bien à cause de cela
(d) cette personne a pensé : cela est bien 


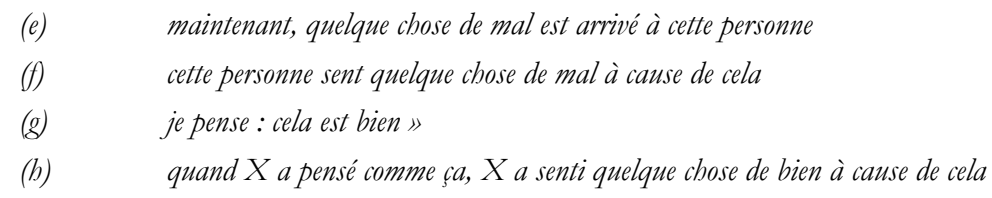

(b) quand $X$ a pensé comme ça, $X$ a senti quelque chose de bien à cause de cela

J'ai fourni une explication allemande aussi bien qu'anglaise et française afin de montrer, une fois de plus, que la métalangue sémantique naturelle n'est pas associée à une langue particulière (quelle qu'elle soit) mais qu'elle est, par définition, indépendante : il y a une MSN allemande, aussi bien qu'une MSN chinoise, une MSN française, et ainsi de suite ; et, qui plus est, ces différentes MSN sont isomorphiques. Cela veut dire qu'une explication formulée en anglais, plutôt que de dépendre du lexique anglais et de la grammaire anglaise, peut être reformulée sans effort dans n'importe quelle autre langue.

Les explications du mot Schadenfreude formulées dans les MSN anglaise et française permettent de présenter de façon précise le sens de ce mot à des locuteurs anglais et français. Puisque ces explications se laissent en outre transposer directement dans n'importe quelle autre MSN, elles peuvent, de façon semblable, présenter le sens de ce mot à des locuteurs d'autres langues. La correspondance précise des explications, qui relient le sens communiqué par un locuteur à l'interprétation d'un tiers, n'est possible qu'à la suite du fait que des mots tels que moi, quelqu'un, penser, sentir, bien et mal (au sens pertinent) ont des équivalents dans toutes les autres langues, et que des combinaisons syntaxiques telles que quelque chose de bien, quelque chose de mal ou quelqu'un d'autre sont attestées de façon universelle. Qui plus est, comme une version allemande de l'explication est elle aussi disponible, on peut voir dans la paraphrase du mot Schadenfreude ci-dessus une réflexion de ce qu'entendent par ce mot des locuteurs allemands - à qui on peut en outre soumettre pour évaluation l'explication proposée.

En résumé, puisqu'il existe un ensemble de primitifs universels lexicalisés dans toutes les langues et une grammaire universelle qui régit la combinaison de ces primitifs, nous avons à notre disposition une mini-langue universelle, ou plutôt un ensemble de mini-langues universelles qui se correspondent et qui sont tout à fait intertraduisibles. Chacune de ces langues constitue une «métalangue sémantique naturelle » ou MSN qui permet d'expliquer aux locuteurs d'une langue quelconque l'ensemble des sens complexes existant dans cette langue, et qui peut servir en outre de passerelle permettant de rendre accessibles à ces mêmes locuteurs les sens propres à d'autres langues et à d'autres cultures. On peut désigner ces mini-langues comme la MSN anglaise, la MSN française, la MSN chinoise, etc., mais sans jamais perdre de vue qu'elles sont isomorphiques et qu'elles constituent en réalité des variantes distinctes d'un système conceptuel unique - la « lingua mentalis » humaine et universelle (cf. Wierzbicka 1980).

Il est possible d'aller plus loin et de recourir aux différentes mini-langues à notre disposition pour rédiger des textes entiers - des textes qui, d'un point de vue sémantique, contrairement aux traductions dont nous avons l'habitude, se correspondent de manière rigoureuse. Afin d'illustrer que, même au niveau textuel, l'équivalence sémantique totale est possible, et que les textes eux-mêmes peuvent être intuitivement intelligibles à des locuteurs ordinaires de la langue retenue, aussi bien que manifestement équivalents au-delà des frontières linguistiques, je reproduirai deux textes assez brefs de mon ouvrage What did Jesus mean? (Wierzbicka 2001). Mon but 
Anna Wierabicka

est de montrer qu'il est possible de les transposer, sans la moindre altération sémantique, en français - et aussi, telle est ma conviction, dans toute autre langue. L'un des textes fournit une explication de la parabole du pharisien et du publicain, tirée de l'évangile de Luc ; l'autre, une explication du concept judéo-chrétien de Dieu :

Le pharisien et le publicain (version anglaise)

a) sometimes people think like this:

b) «I am not like some other people

c) these other people are bad people

d) they do many bad things

e) they don't live with God

f) I am someone good

g) I don't do bad things

b) I do many good things

i) I live with God

j) this is very good》

k) it will be bad for you if you think like this

l) when a person thinks like this this person doesn't live with God

m) it will be good or you if you think like this:

n) «I am not someone good

o) I do many bad things

p) God is someone good

q) I want to live with God»

A l'exception du mot God, auquel je reviendrai dans quelques instants, tous les mots dans cette explication représentent des primitifs sémantiques universels et possèdent dès lors des contreparties correspondantes dans toutes les langues, notamment en français :

a) parfois les gens pensent ainsi :
b) "je ne suis pas comme d'autres gens
c) ces autres gens sont des gens méchants
d) ils font beaucoup de mal
e) ils ne vivent pas avec Dieu
f) je suis quelqu'un de bien
g) je ne fais pas de mal
b) je fais beaucoup de bien
i) je vis avec Dieu
j) cela est très bien 》


k. _e sera mal pour toi si tu penses ainsi

l) quand quelqu'un pense ainsi cette personne ne vit pas avec Dieu

m) ce sera bien pour toi si tu penses ainsi:

n) "je ne suis pas quelqu'un de bien

o) je fais beaucoup de mal

p) Dieu est quelqu'un de bien

q) je veux vivre avec Dieu»

Le mot Dieu est sémantiquement complexe, mais il est possible de l'expliquer à l'aide de primitifs sémantiques, de la façon suivante :

Dieu (version anglaise)

a) God is someone (not something)

b) this someone is someone good

c) this someone is not someone like people

d) there isn't anyone else like this someone

e) this someone exists always

f) everything exists because this someone wants it to exist

g) people exist because this someone wants them to exist

b) this someone exists because this someone exists, not because of anything else

i) this someone lives

Comme toutes les autres explications, celle-ci se laisse directement transposer en français (aussi bien que dans n'importe quelle autre langue) :

Dieu

a) Dieu est quelqu'un (pas quelque chose)

b) ce quelqu'un est quelqu'un de bien

c) ce quelqu'un n'est pas comme les gens

d) il n'y a personne d'autre comme ce quelqu'un

e) ce quelqu'un existe toujours

f) tout existe parce que ce quelqu'un veut que tout existe

g) les gens existent parce que ce quelqu'un veut qu'ils existent

b) ce quelqu'un existe parce que ce quelqu'un existe, non pas à cause d'autre chose

i) ce quelqu'un vit

Quand je parle d'universaux substantiels empiriques, je parle d'éléments tels que QUELQU'UN et QUELQUE CHOSE, ou BIEN et MAL, qui peuvent être transposés dans n'importe quelle autre langue, non seulement à l'état isolé, mais aussi et avant tout dans le contexte de phrases particulières. Si ces phrases respectent les propriétés 
Anna Wierabicka

combinatoires des primitifs (conformément aux principes exposés dans Sens et grammaire universelle), elles peuvent se correspondre sémantiquement à travers toutes les langues, et ce de façon rigoureuse.

\section{Les universaux empiriques du langage et de la pensée}

«Le trait le plus fascinant, peut-être, de l'univers des langues est leur diversité. » Cette assertion, de l'éminent typologiste français Claude Hagège (1985 : 54), auteur de plusieurs travaux descriptifs portant sur un grand nombre de langues diverses parlées en Afrique, aux Amériques, en Asie, en Océanie aussi bien qu'en Europe, du mbum (Cameroun) au chinois, et de l'arabe au comox laamen (Colombie britannique), est de celles que bien des linguistes n'hésiteraient guère à prendre à leur compte.

Pour beaucoup d'autres chercheurs dans le domaine des sciences du langage, une fascination inspirée seulement par la diversité des langues paraît cependant curieusement disproportionnée. Ce qu'ils trouvent plus fascinant encore que la diversité en tant que telle, c'est l'interaction entre la diversité et l'unité essentielle, entre l'éventail de plus en plus large des spécificités linguistiques connues et l'émergence de plus en plus claire d'universaux solides et indiscutables du langage.

A l'instar de plusieurs de ses collègues typologistes, Claude Hagège, sous le coup de la grande diversité des langues, a lui aussi exprimé ses réserves quant à l'existence d'universaux du langage - du moins de «véritables universaux » ou, pour utiliser ses propres mots, d'« universaux de substance ». Il a ainsi fait remarquer qu'il est « évident que dans le monde linguistique, les écarts sont trop accusés pour que des universaux de substance aient quelque apparence de probabilité » (Hagège 1985: 64). Notre confrère continue en produisant et en récusant plusieurs exemples putatifs, dont ceux-ci :

[T] outes les langues ont des adverbes signifiant "toujours" et "seulement" » : cela se trouve infirmé par des langues comme le palau (Micronésie) ou le comox (Colombie britannique), où il s'agit de verbes, dans des structures du type «il-toujours-passé travailler», signifiant «il travaillait toujours». Ou bien : «si les adjectifs de mesure formant couple d'antonymes sont dérivés l'un de l'autre, c'est "petit" qui sera dérivé et "grand" qui sera base» : cela se vérifie souvent, mais il y a des contre-exemples, comme celui du bugis (Célèbes, Indonésie), où «grand» se dit «non petit » (tengbaiccu). Ou enfin: "il existe universellement un nom homme et un verbe voir primaires, c'est-à-dire, étant donné l'importance et la généralité des concepts correspondants, un nom et un verbe qui sont des mots simples non analysables, et non des composés ou dérivés »: assertion démentie par le diegueño (Mexique), où «homme» se dit (...) « celui qui est grand», et par le kalam (Nouvelle-Guinée), où « voir » se dit « (avec les) yeux-percevoir. (Hagège $1985: 65)$.

Peu importe, au sein de la théorie MSN, que les mots pour 'homme' et VOIR soient morphologiquement simples ou complexes, que le mot pour 'toujours' soit un adverbe ou un verbe, et que le mot pour GRAND soit morphologiquement plus ou moins complexe que le mot pour PETIT. Ce qui est bien plus important, c'est que 
toutes les langues possèdent effectivement des mots, ou des éléments comparables tels que des morphèmes ou des phrasèmes, pour 'homme' et VOIR, pour GRAND et PETIT, pour TOUT et TEMPS, mots qui peuvent être insérés dans des phrases canoniques, sans qu'il y ait la moindre différence sémantique identifiable. En français, les mots pour QUELQU'UN et QUELQUE CHOSE sont formellement complexes, et les lexicalisations de QUAND incluent une forme complexe (A UN MOMENT) aussi bien que quelques formes simples (QUAND, MOMENT et FOIS). C'est une question de structure formelle, et les structures formelles sont toujours spécifiques. La grammaire universelle, par contre, est essentiellement une grammaire conceptuelle. Si, à titre d'exemple, TOUJOURS peut être une molécule sémantique universelle, c'est que dans toutes les langues du monde les primitifs conceptuels TOUT et TEMPS peuvent être combinés en vue de constituer une telle molécule. Du point de vue de la sémantique aussi bien que de celui de la grammaire universelle, la façon dont cette molécule ou les primitifs constitutifs de la molécule se réalisent formellement est sans importance.

Ayant passé en revue plusieurs universaux putatifs, Hagège se trouve amené à s'interroger : «Si l'on trouve tant de flagrants démentis aux universaux de substance, » se demande-t-il, "est-ce à dire qu'il faille s'en tenir à des universaux de forme? " (p. 66). La réponse est négative, étant donné que ces soi-disant "universaux de forme » (tels que les conçoit, avant tout, la grammaire générative) sont si éloignés de la réalité des langues que, dans les paroles de l'auteur, ils «peuvent nous apprendre quelque chose (...) sur l'ingéniosité de celui qui les façonne, mais non sur les langues ellesmêmes » (p. 67). En conclusion, Hagège renonce à la quête aux universaux et déclare sa préférence pour l'étude de la diversité des faits linguistiques.

En ce qui me concerne, je partage l'indifférence de mon confrère parisien quant à la recherche d'universaux de forme, c'est-à-dire d'universaux abstraits et sans rapport avec la substance des langues. La recherche d'universaux de substance, par contre, est une tout autre affaire. Il y a de bonnes raisons, me semble-t-il, pour rejeter comme étant excessives les réserves exprimées par Hagège dans son ouvrage de 1985 ; je n'en veux pour preuve que les études empiriques publiées dans les deux volumes de Sens et grammaire universelle (et dans le plus ancien Semantic and Lexical Universals, Goddard et Wierzbicka réd. 1994). Il faut toutefois se rappeler que la quête de véritables "universaux de substance» ne peut se faire que dans le contexte d'une analyse sémantique rigoureuse. Les recherches empiriques des deux dernières décennies ont permis de constater que dans toutes les langues il existe effectivement des mots pour QUELQU'Un et QUELQUE CHOSE, pour GRAND et PETIT, etc. Mais il aurait été impossible de s'en convaincre en l'absence d'une théorie sémantique appropriée et d'une méthodologie efficace.

De façon plus générale, je suis d'accord pour dire que les seuls universaux du langage qui valent la peine d'être répertoriés sont des universaux empiriques; mais je crois aussi qu'une recherche empirique, qu'elle porte sur les universaux du langage ou bien sur la diversité des langues, n’aboutira à des résultats que si le chercheur dispose d'un cadre théorique rigoureux. Grâce à la théorie sémantique MSN développée au cours des trois dernières décennies sur la base de recherches détaillées portant sur des langues très diverses, il est possible de distinguer les universaux authentiques de ceux qui ne le sont pas, et d'établir, par des moyens empiriques, que l'authenticité de certains universaux est simplement irréfutable. En fait, la théorie MSN permet de 
démontrer qu'au sein de leur lexique aussi bien qu'au sein de leur grammaire, toutes les langues partagent un noyau universel, un noyau qui sous-tend l'interprétation, la communication et la traduction humaines. Elle permet en outre de démontrer que ce noyau partagé est comme une mini-langue susceptible de servir de métalangue culturellement neutre, qu'il s'agisse de décrire les langues du monde, ou encore d'étudier la diversité culturelle aussi bien que l'unité psychologique de la race humaine, ou enfin de résoudre certains des problèmes concrets qui se posent dans l'enseignement et dans la communication transculturelle.

J'ai cité, peut-être trop longuement, deux critiques de la quête des universaux du sens et de la grammaire, à savoir Van Brakel et Hagège. L'un d'eux visait de façon très spécifique la version MSN de cette quête. J'espère que l'on me pardonnera de redresser brièvement l'équilibre, en citant un partisan non seulement de la quête d'universaux sémantiques en général, mais du projet MSN en particulier.

L'anthropologue Roy D'Andrade (2001 : 246) vient de donner son aval à la théorie et à la pratique MSN en écrivant que la métalangue sémantique naturelle « offre le potentiel de coucher tout ce qui est complexe en langue ordinaire et de traduire des concepts d'une langue à l'autre sans perte ni distorsion au niveau du sens ». Ayant rappelé que "l'idée du développement d'une métalangue universelle a souvent été proposée à la fois par des philosophes et des linguistes », D'Andrade décrit l'approche MSN comme «l'exécution à ce jour la plus rigoureuse et la plus complète de ce programme » (voir aussi Jones 1999 ; Shweder à paraitre).

D'Andrade observe que « si on entend par culture un ensemble d'idées, de sens, de connaissances, d'interprétations partagées, ces idées partagées doivent se composer soit de termes primitifs indéfinissables soit de conglomérats de termes primitifs » (p. 248). Les termes universaux identifiés au sein du programme MSN, dit l'auteur, sont « analogues aux atomes du monde physique », analogie qu'il développe ensuite de la façon suivante :

Parmi les innombrables combinaisons de ces termes qui donnent lieu aux phrases correspondant aux idées / sens / connaissances / interprétations possibles d'un individu, certaines sont culturelles - c'est-à-dire partagées intersubjectivement par des collectivités au sein d'une société. Tout comme plus d'une centaine de types d'atomes se combinent pour former plus de 20 millions de types de molécules, les cinquante et quelques concepts universaux se combinent pour former des centaines de milliers d'idées. Voilà qui met l'anthropologue qui connaît la métalangue sémantique naturelle, et qui est capable de l'utiliser, dans la même position que le chimiste qui connaît ses atomes - la plupart des objets du monde sont des molécules, et ce sont leurs propriétés que l'on cherche à mieux connaitre. La connaissance des atomes est chose utile pour le chimiste dans la mesure où elle permet de comprendre l'essence des molécules.

L'analogie entre la sémantique MSN et la chimie, établie par D’Andrade, a été creusée un peu plus dans un article récent de mon collègue et corédacteur de Sens et grammaire universelle, Cliff Goddard. Dans cet article, Goddard approfondit le parallélisme entre la table MSN des primitifs sémantiques et la table périodique des éléments de Mendeleïev en explorant la question de savoir «à quel point les propriétés 
[grammaticales] pertinentes se distribuent de manière systématique sur la totalité de l'inventaire des primitifs, et dans quelle mesure elles s'alignent et dépendent les unes des autres à la façon des éléments chimiques ». C'est un développement nouveau et excitant du programme MSN. Cependant, même dans sa forme actuelle quelque peu instructurée, la table des primitifs sémantiques a permis de tracer des voies nouvelles dans le domaine de la grammaire universelle et de mettre à la disposition de ceux qui cherchent à articuler des sens complexes culturellement spécifiques, et à les rendre accessibles à des tiers, un instrument efficace, une véritable métalangue - à savoir la métalangue sémantique naturelle.

Malheureusement l'approche MSN, si bien comprise par l'anthropologue D'Andrade, vient de tomber victime du regard déformant du linguiste suisse Patrick Sériot (2004). Une discussion exhaustive de ses observations étant impossible dans les limites du présent article, je renvoie le lecteur à la réplique détaillée que j’ai publiée ailleurs (Wierzbicka, à paraitre) et je me contente dans ce qui suit de faire deux remarques.

Pour commencer, Sériot détecte dans l'approche MSN «un présupposé unanimiste jamais explicité : tous les gens parlant la même langue pensent de la même façon ». Pareil "présupposé », dont l'absurdité saute aux yeux, est totalement contraire à ce qui a été avancé dans des dizaines de publications pertinentes : à savoir que les « mots clés » d'une langue particulière, des mots culturellement saillants tels que sud'ba en russe ou fairness en anglais, favorisent certaines façons de penser, sans toutefois les imposer. En dernière analyse, le fait même que toutes les notions humaines, quelles qu'elles soient, constituent des configurations distinctes d'un ensemble unique de primitifs conceptuels implique que la langue utilisée au sein d'une communauté donnée ne saurait déterminer les façons de penser de ceux qui la parlent.

Ailleurs, Sériot soulève une question rhétorique: "Si la totalité du sens préexiste dans le lexique et la grammaire (...), comment peut-on dire quoi que soit de neuf ? ». C'est un autre présupposé absurde, attribué à l'approche MSN mais contraire à ce qu'elle proclame : un ensemble universel de primitifs conceptuels, qui se manifeste dans toutes les langues, permet d'engendrer un nombre infini de nouveaux sens et de nouvelles idées. Ainsi que l'explique D'Andrade, «Tout comme plus d'une centaine de types d'atomes se combinent pour former plus de 20 millions de types de molécules, les cinquante [ou plutôt soixante, A.W.] et quelques concepts universaux se combinent pour former des centaines de milliers d'idées ».

Il est malencontreux que les deux volumes clés de l'approche MSN, sortis en 2002 sous le titre de Sens et grammaire universelle, ne figurent même pas dans la bibliographie de Sériot. C'est avant tout à ces volumes, qui reproduisent les paroles de D'Andrade, que je me permets de renvoyer ceux qui voudraient se familiariser avec les tenants et aboutissants de l'approche MSN, sa base empirique et ses résultats concrets. Je conclurai en reproduisant, en français, les derniers paragraphes de Sens et grammaire universelle:

Si la métalangue sémantique naturelle est une entité factuelle, il doit être possible de mener à bon terme sa description exhaustive et l'identification de ses propriétés. Le présent ouvrage touche à sa fin, mais notre tâche n'est pas tout à fait terminée. Je crois néanmoins que l'essentiel a été accompli. Avec un peu de chance, l'objectif sera réalisé avant la fin de la décennie. Mais ce ne sera guère la fin de l'histoire, au contraire. Tout comme la découverte de nouveaux éléments chimiques et de leurs 
Anna Wierzbicka

propriétés combinatoires a ouvert de nouvelles perspectives en chimie, il faut s'attendre à ce que la description exhaustive de la métalangue sémantique naturelle ouvre de nouvelles perspectives dans l'étude du langage, de la pensée et de la culture. Il reste d'importantes découvertes à faire dans des domaines aussi divers que la structuration sémantique spécifique du vocabulaire et de la grammaire (la lexicogrammaire), la structure et les pratiques du discours, la communication non verbale, et nombre d'autres phénomènes culturels et sémiotiques. Il faut s'attendre en outre à ce que de nouvelles pistes se présentent non seulement dans le domaine de l'anthropologie et de la psychologie cognitives (voir D'Andrade 2001) et dans celui de la psychologie évolutionniste (voir Jones 1999), mais aussi dans un nombre croissant de domaines appliqués tels que la communication transculturelle, l'enseignement des langues, les cours de droit et la thérapie du langage.

Le vingtième siècle n'était pas propice à l'étude du sens. Le vingt et unième, on le devine, sera très différent. (Goddard 2002b : 315) 


\section{RÉFÉRENCES BIBLIOGRAPHIQUES}

D'ANDRADE, R., 2001, "A cognitivist's view of the units debate in cultural anthropology ». Cross-Cultural Research 35(2) : 242-257.

GODDARD, C., 1997, «Contrastive semantics and cultural psychology : 'surprise' in Malay and English ». Culture and Psychology 3(2) : 153-181.

GodDard, C., 1998, Semantic Analysis : A practical introduction. Oxford, Oxford University Press.

GODDARD, C., 2001, « Lexico-semantic universals : A critical overview ». Linguistic Typology 5(1) : 1-66.

GODDARD, C., 2002a, «Semantic primes and universal grammar in Malay (Bahasa Melayu)». In : Goddard et Wierzbicka (réd.), vol. 1, 87-172.

GODDARD, C., 2002b, «The ongoing development of the NSM research program». In: Goddard et Wierzbicka (réd.), vol. 2, 301-321.

GODDARD, C., à paraitre, «A systematic table of semantic elements : Some preliminary remarks ».

GodDARD, C. et WierzBickA, A. (réd.). 1994. Semantic and Lexical Universals : Theory and empirical findings. Amsterdam, John Benjamins.

Goddard, C. et WierzBickA, A., 2002, Meaning and Universal Grammar: Theory and empirical findings. 2 volumes. Amsterdam : John Benjamins.

Hagege, C., 1985, L’homme de paroles : Contribution linguistique aux sciences humaines. Paris, Fayard.

JONES, D., 1999, «Evolutionary psychology ». Annual Review of Anthropology 28 : 553-575.

Peeters, B. (réd.), 2006, Semantic Primes and Universal Grammar : Empirical evidence from the Romance languages. Amsterdam, John Benjamins.

PINKER, S. 1994. The language instinct. How the mind creates language. New York, William Morrow. Trad. française (1999), L’instinct du langage (Paris, Odile Jacob).

PINKER, S., 1997, How the mind works. New York, Norton.

SHWEDER, R., à paraittre, «Deconstructing the emotions for the sake of comparative research ».

VAN BRAKEL, J., 2002, «Chromatic language games and their congeners ». In Saunders Barbara et Jaap Van Brakel (réd.), Theories, Technologies, Instrumentalities of Color: Anthropological and historiographic perspectives. Lanham, Maryland, University Press of America: 147-168.

WIERZBICKA, A., 1972, Semantic Primitives. Frankfurt am Main, Athenäum (Linguistische Forschungen, 22).

WierZBiCKA, A., 1980, Lingua Mentalis : The semantics of natural language. Sydney, Academic Press.

WierzBickA, A., 1996, Semantics : Primes and universals. Oxford, Oxford University Press.

WiERZBICKA, A., 1998, « Anchoring linguistic typology in universal human concepts ». Linguistic Typology 2(3) : 141-194.

WierzBickA, A., 2001, What did Jesus mean? Explaining the Sermon on the Mount and the Parables in simple and universal human concepts. New York, Oxford University Press.

WierzBickA, A., 2002, «Semantic Primes and Universal Grammar in Polish ». In: Goddard et Wierzbicka (réd.), vol. 2 : 65-145. 
